\title{
Patterns of airway disease and the clinical diagnosis of asthma in the Busselton population
}

\author{
A.W. Musk*,\#, M. Knuiman*\#, M. Hunter*, J. Hui*,+,\$, L.J. Palmer*,f, J. Beilby*,, \\ M. Divitini ${ }^{\#}$, S. Mulrennan and A. James ${ }^{\star * \star *}$
}

ABSTRACT: The aim of this study was to examine how objective measures related to lung function cluster in the general population and how the patterns relate to asthma and bronchitis as diagnosed by a doctor (DDA and DDB, respectively).

A cross-sectional survey of an age-stratified random general population sample of 1,969 adults from the electoral register of Busselton (Australia) was performed in 2005-2007. Respiratory symptoms, DDA ever, DDB ever, recent wheezing and smoking history, together with anthropometric measurements, forced expiratory volume in 1 s (FEV1) and forced vital capacity (FVC), methacholine challenge or bronchodilator response, exhaled nitric oxide (eNO), skin-prick tests to common allergens, and blood eosinophil and neutrophil counts were studied. Cluster analysis (variables sex, age, atopy, FEV 1 \% predicted, FEV1/FVC, airway hyperresponsiveness, eNO, log eosinphil count, log neutrophil count and body mass index) was used to identify phenotypic patterns.

Seven clusters (subjects with DDA and DDB, respectively) were identified: normal males ( $n=467 ; 7$ and 13\%), normal females $(n=477 ; 12$ and $18 \%)$, obese females $(n=250 ; 16$ and $28 \%)$, atopic younger adults $(n=330 ; 21$ and 17\%), atopic adults with high eNO ( $n=130 ; 30$ and 25\%), atopic males with reduced $\mathrm{FEV}_{1}(\mathrm{n}=103 ; 33$ and $32 \%)$ and atopic adults with bronchial hyperreactivity ( $n=212 ; 40$ and $26 \%$ ).

The clinical diagnosis of asthma (ever) and bronchitis (ever) is not specific for any of the clustering patterns of airway abnormality.

\section{KEYWORDS: Asthma, clinical diagnosis}

ince the international Ciba Guest Symposium in 1959 [1] and the 1962 statement of the American Thoracic Society [2], asthma has generally been defined in physiological terms as variable airflow obstruction, emphysema has been defined in morphological terms and bronchitis in clinical terms. The conventional clinical entities of asthma and chronic obstructive pulmonary disease (COPD) are heterogeneous conditions with a large degree of overlap and encompass virtually all patients with obstructive spirometry who have no other proven specific airway disease processes. This heterogeneity exists with respect to clinical, physiological and pathological factors, and is observed both between individuals and within individuals over the course of their lives. The conditions are complex disorders and thought to be due to the interaction of multiple genetic and environmental factors whose relative contributions to disease expression probably vary with age and between different populations and different geographical locations. This degree of heterogeneity has led to the idea that multiple distinct pathophysiological entities underlie the clinical entities [3-5].

The conflation of diverse processes into clinical labels may have contributed to the difficulties that have been encountered in understanding these diseases, especially studies of genetic epidemiology [6], and of novel treatments that may only target one molecular pathway, therefore, being effective in only those patients with a particular subphenotype [7].

A variety of asthma phenotypes have been recognised [8-10] and it has been suggested that better definitions of asthma subtypes should form the basis of treatment options for patients that have, in the past, been classified mainly by clinical severity [11]. This approach is yet to be

\section{AFFILIATIONS}

*Busselton Population Medical Research Foundation, Sir Charles Gairdner Hospital,

'Depts of Respiratory Medicine, and **Pulmonary Physiology, Sir Charles Gairdner Hospital,

${ }^{*}$ Schools of Population Health, and §Pathology and Laboratory Medicine, University of Western Australia, and +PathWest, Queen Elizabeth II Medical Centre, Nedlands, Australia. ${ }^{f}$ Genetic Epidemiology and Biostatistics Platform, Ontario Institute for Cancer Research, Toronto, ON, Canada.

\section{CORRESPONDENCE}

J. Hui

University of Western Australia

Molecular Genetics

PathWest

J Block

Queen Elizabeth II Medical Centre

Nedlands

WA 6009

Australia

E-mail: jhui@cyllene.uwa.edu.au

Received:

July 012010

Accepted after revision:

March 232011

First published online:

May 122011

European Respiratory Journal Print ISSN 0903-1936 Online ISSN 1399-3003 
widely adopted for clinical use, since accurate characterisation of different phenotypes is difficult and not standardised. Similar concerns regarding the diagnosis and management of COPD have been expressed [12].

In an effort to improve phenotype characterisation, statistical analyses of patient populations have been explored $[5,13]$. This approach has been used in patients diagnosed with asthma or COPD [11, 14-17]. To a large extent, these populations have already been selected based on clinical severity, clinical setting or physiological abnormality based on lung function testing. Such selection introduces bias (such as diagnostic and recall bias) in the definition of phenotypes, and ignores the crossover of symptoms and abnormalities of airway function that occur between various airway diseases.

The aim of the present study was to examine the clustering of objective measures of airway disease in the general population and determine how well these patterns relate to the conventional diagnostic entities of asthma and bronchitis (as diagnosed by a doctor), tobacco smoking and respiratory symptoms. The research hypothesis was that objective measures of measurable airway-related attributes in the general population define discrete phenotypic clusters, all of which include "doctor-diagnosed asthma" (DDA) and are more homogeneous than conventional diagnostic labels of asthma, chronic obstructive lung disease (COPD) and bronchitis.

\section{MATERIALS AND METHODS \\ Subjects}

An age-stratified random sample of 2,932 adults (63\% of those contacted) identified from the electoral register (registration to vote is compulsory in Australia) for the district of Busselton in Western Australia participated in a prevalence study of respiratory disease conducted between July 2005 and July 2007. Due to missing data for some clinical measurement variables (each variable had 100-300 missing values) there was a total of 1,979 adults who had all required data for this analysis. The participants were predominantly Caucasian with some having Asian ancestry. No Aboriginal people took part in the survey.

\section{Methods}

Written informed consent was obtained and the study was approved by the Human Research Ethics Committee of the University of Western Australia (Nedlands, Australia). A mailed questionnaire included respiratory symptoms, smoking and previous illnesses based on the British Medical Research Council Respiratory Questionnaire [18]. The diagnosis of asthma (DDA-ever) was taken from an affirmative response to the question, "Has a doctor ever told you that you have asthma?" Doctor-diagnosed bronchitis (DDB)-ever was defined as a positive response to the question, "Has a doctor ever told you that you have bronchitis?" Recent wheeze was defined as a positive response to the question, "Has your chest ever made a wheezing or whistling sound? If yes, in the last 12 months?" Height $(\mathrm{cm})$ and weight $(\mathrm{kg})$ were measured with no shoes on and without wearing coats/jumpers. Body mass index (BMI) was calculated as $\mathrm{kg} \cdot \mathrm{m}^{-2}$. Ever-smokers had smoked at least one cigarette per day or $50 \mathrm{~g}$ of tobacco per month for $\geqslant 1 \mathrm{yr}$. For analysis, smoking history was scored as never-smoker, exsmoker or current smoker. The latter group was split into two according to the pack-yrs of smoking ( $<5$ and $>5$ pack-yrs), with a pack-yr defined as smoking the equivalent of one pack (20 cigarettes) per day for a year. Cough and phlegm were recorded if subjects reported cough and phlegm production on most days for $\geqslant 3$ months of the year.

Forced expiratory volume in $1 \mathrm{~s}$ (FEV1) and forced vital capacity (FVC) were measured using Medgraphics CPFS/D USB pneumotach spirometers and recorded with BreezeSuite 6.2 software (both Medical Graphics Corporation, St Paul, MN, USA). Spirometers were calibrated daily using a 3-L syringe and corrected for room temperature, humidity and barometric pressure. Forced expiratory manoeuvres were performed with the subject seated and wearing a nose clip, and were repeated up to a maximum of eight efforts until three readings of FEV1 and FVC, reproducible to at least $200 \mathrm{~mL}$ [19], were obtained, although attempts were made to achieve reproducibility of $<75 \mathrm{~mL}$ [20]. The highest values for FEV1 and FVC were recorded. The best FEV1 and FVC were expressed as percentages of the predicted values [21].

Airway responsiveness was measured by calculating the dose of inhaled methacholine chloride that provoked a $20 \%$ fall in FEV1 (PD20). Participants inhaled normal saline and then increasing doses of methacholine $\left(0.03-4 \mathrm{mg} \cdot \mathrm{mL}^{-1}\right)$ via a handheld, dosimeter-driven nebuliser (Model 45; DeVilbiss, Health Care, Somerset, PA, USA), and FEV1 was measured at 30 and 90 s. The test was stopped if there was a $>20 \%$ fall in FEV1 from the post-saline FEV1, the maximal dose was delivered or the subject was unwilling to continue. Participants were excluded from the methacholine challenge if they were pregnant or breastfeeding, were taking $\beta$-adrenergic blocker medication or had used a long acting $\beta_{2}$-agonist aerosol in the preceding $48 \mathrm{~h}$. Because of the risk of causing severe/unpleasant symptoms, participants with an initial FEV1 of $<60 \%$ predicted did not receive methacholine but were given salbutamol $(200 \mu \mathrm{g})$ administered from a metered-dose inhaler via a spacer and spirometry was repeated after $15 \mathrm{~min}$. Airway hyperresponsiveness was defined as a PD20 of $<4 \mu \mathrm{mol}$ of methacholine or a $>15 \%$ increase in FEV1 following salbutamol, as both have been shown to similarly define hyperresponsiveness [22].

Forearm skin-prick responses to inhaled common allergens (rye grass pollen, grass mix, house dust mite (Dermatophagoides pteronyssinus and D. farinae), cat pelt, dog dander, Alternaria tenius, Aspergillus fumigatus and mould mix; Hollister-Stier Laboratories, Spokane, WA, USA) were assessed. Histamine $\left(10 \mathrm{mg} \cdot \mathrm{mL}^{-1}\right)$ and glycerine were used as positive and negative controls, respectively. Weal size (average of the long axis and its perpendicular in $\mathrm{mm}$ ) was measured after $10 \mathrm{~min}$ for the positive control and after $15 \mathrm{~min}$ for the allergens. Atopy was defined as any allergen weal of $\geqslant 3 \mathrm{~mm}$ greater than the negative control. Exhaled nitric oxide (eNO) was measured prior to spirometry and methacholine challenge using a calibrated chemiluminescence analyser with online measurement of single exhalations (Niox; Aerocrine, Solna, Sweden) according to a standard protocol [23]. Absolute neutrophil and eosinophil counts were performed on a venous blood sample collected at the time of the survey.

K-means cluster analysis (SAS Version 9.2; SAS Institute Inc., Carey, NC, USA) was used to divide participants into homogeneous groups. The quantitative variables in the cluster 
analysis were: sex, age, atopic status, bronchial hyperreactivity (BHR), FEV1\% pred and FEV1/FVC, eNO, blood eosinophils, blood neutrophils and BMI. The logarithm of the neutrophil and eosinophil counts was used in the analysis as they showed skewed distributions. Initially, a cluster model with 40 clusters was fitted to the data to identify outliers (subjects who were allocated to a cluster of fewer than five people) and after removing 10 outlying subjects, there were a total of 1,969 subjects in the main cluster analysis. The number of clusters was based on examining the change in the ratio of the between- to within-cluster variation as more clusters were added, consideration of the size of the resulting clusters ( $\geqslant 100$ subjects) and the stability and consistency of the cluster patterns when analysed separately for sex and smoking groups. Based on these considerations, the seven-cluster model emerged as the most appropriate. For the standardised clustering variables, the total SD was 1.0 (i.e. for the whole sample as a single cluster) and the within-cluster SD declined steadily from 0.94 for the two-cluster model to 0.79 for the seven-cluster model, and thereafter declined more slowly as the number of clusters was increased. The independent variables selected for analysis were chosen to reflect putative mechanisms and measurable clinical characteristics associated with common airway disease phenotypes avoiding those that tend to measure the same thing [16]. It was not possible to determine whether some variables represented epiphenomena given the poor understanding of the fundamental mechanisms of airway diseases.

\section{RESULTS}

Approximately $50 \%$ of the participants were female (table 1). The mean age was $54 \mathrm{yrs}$, the mean BMI was $27 \mathrm{~kg} \cdot \mathrm{m}^{-2}, \sim 50 \%$ were atopic, $12 \%$ had $\mathrm{BHR}, 51 \%$ had never smoked, $38 \%$ were ex-smokers (with two-thirds having smoked $>5$ pack-yrs) and $12 \%$ were current smokers (with three-quarters having already smoked $>5$ pack-yrs), $18 \%$ reported DDA, $24 \%$ reported recent wheeze and 20\% reported DDB.

The seven clusters that emerged appeared naturally to fit groups recognisable in the general population (table 1). Cluster 1 (labelled "normal males") with 467 subjects consisted of males with normal levels of all measured attributes of whom $6.9 \%$ had ever been told by a doctor that they had asthma, $15 \%$ had recent wheeze and $13.3 \%$ bronchitis. Cluster 2 (labelled "normal females") with 477 subjects consisted of females with normal levels of all measured attributes of whom $11.7 \%$ had been told by a doctor that they had ever had asthma, 12.8\% had recent wheeze and $18.4 \%$ bronchitis. Cluster 3 (labelled "obese females") with 250 subjects were mainly overweight females (mean BMI $33.7 \mathrm{~kg} \cdot \mathrm{m}^{-2}, 86 \%$ female) with near normal measured attributes of whom $16.4 \%$ had ever been told by a doctor that they had asthma, 25.6\% had recent wheeze and $28 \%$ bronchitis. Cluster 4 (labelled "atopic younger") with 330 subjects comprised mainly younger adults (mean age $40 \mathrm{yrs}$ ) who were atopic $(70 \%)$ but otherwise had normal measured attributes and $20.9 \%$ had ever been told by a doctor that they had asthma, $26.7 \%$ had recent wheeze, and $17.3 \%$ bronchitis. Cluster 5 (labelled "atopic with high eNO") with 130 subjects was characterised primarily by being atopic (86.2\%) with elevated levels of eNO (mean $56.1 \mathrm{ppb}$ ) but also with a higher prevalence of BHR (16.2\%) and eosinophilia of whom $30 \%$ had ever been diagnosed with asthma by a doctor, $33.8 \%$ had recent wheeze and $24.6 \%$ with bronchitis. Cluster 6 (labelled "atopic males with poor FEV1") with 103 subjects contained mainly older males ( $82.5 \%$ male, mean age $70 \mathrm{yrs}$ ), $79.7 \%$ of whom had ever smoked and whose lung function was impaired (mean $\mathrm{FEV} 1 \%$ pred $66.7 \%$ ) with $33 \%$ having been told by a doctor that they had asthma, 54.4\% had recent wheeze, and $32 \%$ bronchitis. Cluster 7 (labelled "atopic with BHR") with 212 subjects comprised exclusively adults with BHR (100\%) and with a high prevalence of atopy $(70.3 \%)$ but near normal lung function and $39.6 \%$ of whom had been told by a doctor that they had asthma, 38.2\% had recent wheeze, and $25.9 \%$ bronchitis.

Although we have assigned labels to clusters based on the principal distinguishing variables, the other variables are also an integral part of the definition of the clusters as every cluster variable was significantly different across the seven clusters $(p<0.0001)$ and was still significantly different $(p<0.0001)$ across the subset of clusters which did not have that variable as a principal distinguishing variable. To illustrate, BMI is a principal distinguishing variable for cluster 3 ("obese females") and BMI still varies significantly across the other six clusters.

The prevalence rates of DDA were not evenly distributed across the groups $(\mathrm{p}<0.0001)$ with clusters 1 and 2 significantly less than clusters 3 and 4 , which were also significantly less than clusters 5, 6 and 7 . The prevalence of recent wheeze also varied across clusters $(\mathrm{p}<0.0001)$ with a similar ordering to DDA with clusters 1 and 2 having the lowest prevalence, clusters 3 and 4 next lowest, and 5, 6 and 7 the highest prevalence. However, in contrast to DDA, cluster 6 had significantly higher prevalence of recent wheeze than clusters 5 and 6 . Thus, it can be seen that DDA and recent wheeze did not segregate clearly with any particular cluster profile. Current smoking and the prevalence of cough/phlegm also varied significantly between groups (table 1) but again did not segregate with any particular cluster. However, smoking (and in particular ever having smoked at least 5 pack-yrs) clearly segregated with cluster $6(\mathrm{p}<0.001)$, in which one-third reported being told by their doctor that they had asthma, a similar number had bronchitis and over half had recent wheeze.

The prevalence rates of DDA and DDB appeared to be closely related in the various clusters (fig. 1), and the prevalence of DDA was also closely related to that of cough and phlegm (fig. 2). The same was true for recent wheeze (figs 3 and 4). It is evident that there was a tendency for clusters that had a higher prevalence of diagnosed bronchitis and higher prevalence of cough/phlegm to have higher prevalence of diagnosed asthma and recent wheeze. These strong relationships suggest that none was clearly distinguishable from the others in a general population despite the extensive phenotyping that was attempted. The atopic clusters tended to have higher prevalence of all four conditions.

\section{DISCUSSION}

Previous studies of clustering of airway phenotypes have included patients with disease of clinical severity and have not comprised members of the general population. Recently, there have been moves to dissect phenotypes of asthma and COPD that may have implications for treatment or causality. There is increasing evidence in adult patients with asthma that there are distinct groups based on inflammatory profiles of induced sputum and that these may respond to different forms of 
TABLE 1 Subject characteristics: overall and by cluster (phenotypic pattern)

\begin{tabular}{|c|c|c|c|c|c|c|c|c|}
\hline & \multicolumn{8}{|c|}{ Cluster } \\
\hline Total $\mathbf{n}$ & 467 & 477 & 250 & 330 & 130 & 103 & 212 & 1969 \\
\hline Age yrs & $61 \pm 15$ & $54 \pm 17$ & $58 \pm 15$ & $40 \pm 13$ & $48 \pm 19$ & $70 \pm 12$ & $53 \pm 18$ & $54 \pm 17$ \\
\hline Atopic status \% positive & 35.1 & 36.7 & 28.4 & 70.0 & 86.2 & 63.1 & 70.3 & 49.1 \\
\hline BHR \% positive & 0.0 & 0.0 & 0.4 & 0.0 & 16.2 & 12.6 & 100.0 & 12.5 \\
\hline FEV $1 \%$ pred & 101.4 & 99.7 & 94.2 & 99.8 & 97.2 & 66.7 & 88.6 & 96.3 \\
\hline $\mathrm{FEV}_{1 / \mathrm{FVC}}$ & 76.4 & 79.2 & 80.3 & 81.1 & 76.8 & 56.1 & 74.4 & 77.1 \\
\hline \multicolumn{9}{|l|}{ Nonclustering variables } \\
\hline DDA & 6.9 & 11.7 & 16.4 & 20.9 & 30.0 & 33.0 & 39.6 & 18.0 \\
\hline Recent wheeze & 15.0 & 12.8 & 25.6 & 26.7 & 33.8 & 54.4 & 38.2 & 23.6 \\
\hline Cough/phlegm & 22.7 & 15.7 & 20.0 & 25.8 & 22.3 & 38.8 & 27.4 & 22.5 \\
\hline $\mathrm{DDB}$ & 13.3 & 18.4 & 28.0 & 17.3 & 24.6 & 32.0 & 25.9 & 20.2 \\
\hline Never smoked & 45.8 & 60.2 & 50.8 & 49.1 & 66.9 & 20.4 & 46.7 & 50.6 \\
\hline Ex-smoker $<5$ pack-yrs & 11.3 & 16.1 & 9.6 & 10.6 & 8.5 & 7.8 & 12.7 & 11.9 \\
\hline Ex-smoker $>5$ pack-yrs & 36.0 & 17.2 & 26.0 & 16.4 & 20.0 & 58.3 & 24.5 & 25.7 \\
\hline Current smoker $<5$ pack-yrs & 0.6 & 1.5 & 1.2 & 7.3 & 2.3 & 0.0 & 2.8 & 2.3 \\
\hline Current smoker $>5$ pack-yrs & 6.2 & 5.0 & 12.4 & 16.7 & 2.3 & 13.6 & 13.2 & 9.3 \\
\hline
\end{tabular}

Data are presented as $\%$ or mean \pm SD, unless otherwise stated. eNO: exhaled nitric oxide; FEV1: forced expiratory volume in 1 s; BHR: bronchial hyperreactivity; \% pred: \% predicted; FVC: forced vital capacity; BMI: body mass index; DDA: doctor-diagnosed asthma; DDB: doctor-diagnosed bronchitis.

treatment [24, 25]. Using cluster analysis, HALDAR et al. [11] examined asthmatics from an ambulatory care group and another from a hospital specialty clinic setting and found that both groups segregated according to inflammatory markers suggesting that it may be possible to identify groups of patients that respond to different forms of treatment. More recently, MOore et al. [26] examined patients from a severe asthma cohort using cluster analysis and identified five clusters based on atopy, asthma severity and healthcare utilisation. Notably, this study and that of HALDAR et al. [11] identified a cluster of overweight females included in their asthma groups, similar to that seen in the present study. WeATHERALL et al. [17] used cluster analysis to examine 175 subjects with airflow obstruction from a general population sample and were able to identify five clusters based on degree of airflow obstruction, symptoms and markers of emphysema. PILLAI et al. [15] examined features of asthma patients aged 735 yrs that could be useful in identifying important genetic and environmental contributors, and found that baseline pulmonary function, allergen sensitisation, self-reported allergies, symptoms of rhinitis and symptoms of asthma were important. Factors scored as quantitative traits appeared to be better phenotypes for epidemiological and genetic analyses than categories derived from the presence or absence of combinations of positive skin tests or elevated immunoglobulin E. PATEL et al. [27] showed that computed tomography scan measures of airway wall thickness and emphysema independently contributed to airflow obstruction and had independent familial aggregations, suggesting that different inherited factors contribute to airway disease phenotypes. Thus, these studies, undertaken in groups of patients defined by lung function, symptoms or diagnostic labels, show that several distinct phenotypes may exist within the framework of these "diagnostic labels".

The present study was designed differently from other cluster analyses $[3,11,12,14]$ as the aim was to examine how airway disease-related physiological measures in a general population sample cluster without regard to clinical findings/disease status and to determine how the entities of DDA or DDB and recent wheezing segregate within these clusters. Therefore, it was not constrained by preconceived notions of normality and abnormality or clinical diagnostic entities but relied on objective findings that are not dependent on clinical judgement. The results of the study demonstrate that DDA and DDB are distributed across all seven clusters of airway-related phenotypes. 


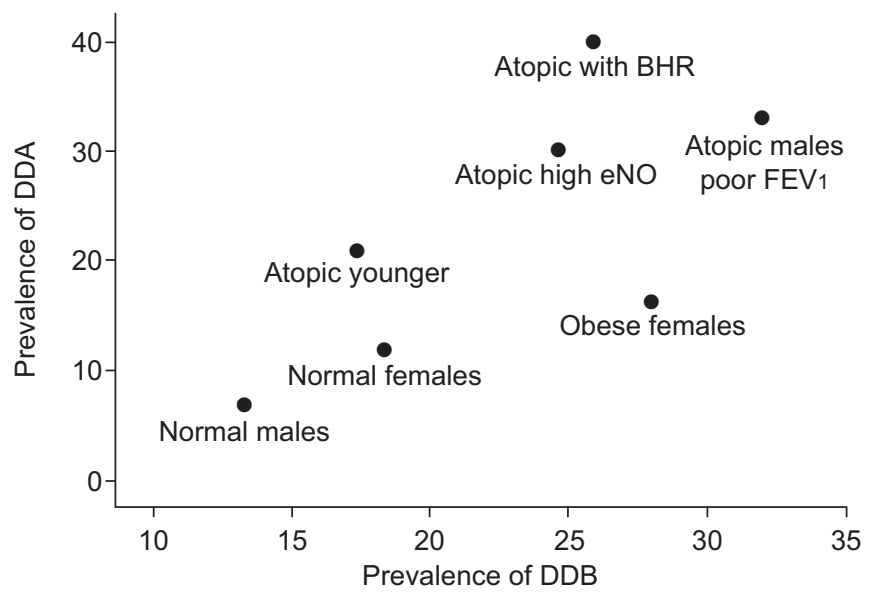

FIGURE 1. Patterns in prevalence of doctor-diagnosed asthma (DDA) and doctor-diagnosed bronchitis (DDB) across clusters. BHR: bronchial hyperreactivity; FEV1: forced expiratory volume in $1 \mathrm{~s}$; eNO: exhaled nitric oxide.

Cluster analysis was utilised for this study because it achieves segregation of people into groups with homogeneous disease manifestations and has recently been suggested as an appropriate method for determination of airway phenotypes [5]. The method used to find clusters (K-means cluster analysis) was the same as in the study by HALDAR et al. [11]. However, other approaches, such as hierarchical methods, have also been suggested. For comparison purposes, we also used a hierarchical approach to the best seven-cluster model and found that five of the cluster types from our K-means approach (1: normal males, 2: normal females, 5: atopic with high eNO, 6: atopic males with poor FEV1, and 7: atopic with BHR) consistently appeared but with cluster types 3 (obese females) and 4 (atopic younger) sometimes slightly modified and with some mixing into "normal" clusters 1 and 2. For example, under the hierarchical method of WARD [28], clusters 1-4 were normal males, normal females, atopic males and atopic females, respectively. Thus, the clinical or disease phenotypic clusters (5-7) emerged consistently in both clustering approaches.

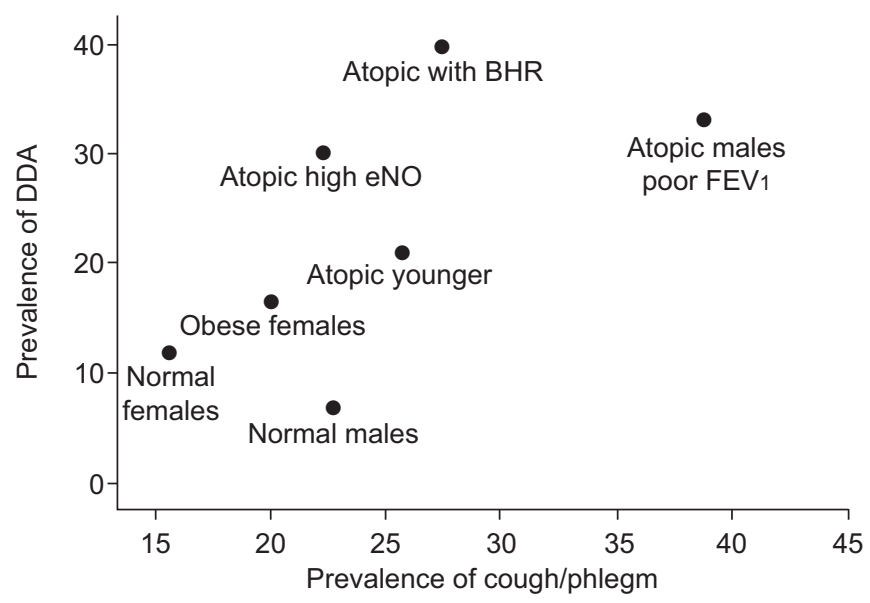

FIGURE 2. Patterns in prevalence of doctor-diagnosed asthma (DDA) and cough/phlegm across clusters. BHR: bronchial hyperreactivity; FEV1: forced expiratory volume in $1 \mathrm{~s}$; eNO: exhaled nitric oxide.

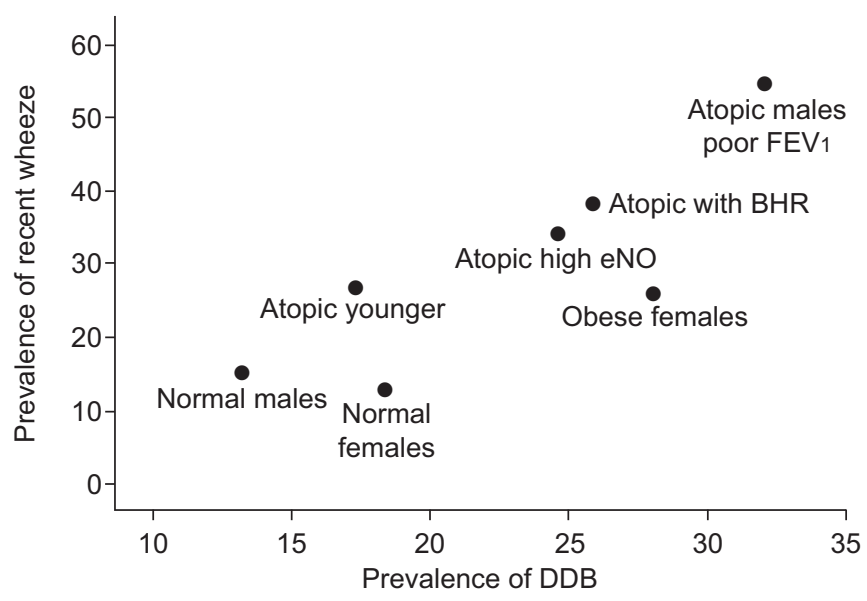

FIGURE 3. Patterns in prevalence of recent wheeze and doctor-diagnosed bronchitis (DDB) across clusters. BHR: bronchial hyperreactivity; FEV1: forced expiratory volume in $1 \mathrm{~s}$; eNO: exhaled nitric oxide.

Furthermore, the pattern of the prevalence of DDA and recent wheeze was essentially unchanged across the cluster types. This suggests that the findings are robust against different statistical methods to find clusters.

We chose objective and quantitative indices related to airway behaviour in a general population sample of adults to examine the patterns of clustering. The use of relatively homogeneous clusters of airway pathophysiology rather than conventional clinical entities such as "asthma" may assist future studies in identifying aetiological factors that contribute to the specific pathways involved in these phenotypic clusters. The approach, however, cannot take into account environmental factors, such as smoking or allergen exposure. This represents the difficulty of diagnosing asthma where the underlying pathology renders the subject susceptible to variable symptoms, which are probably related to environmental exposures. Therefore, symptoms may vary while the "disease process" remains present. For this reason, we examined the association of a cumulative

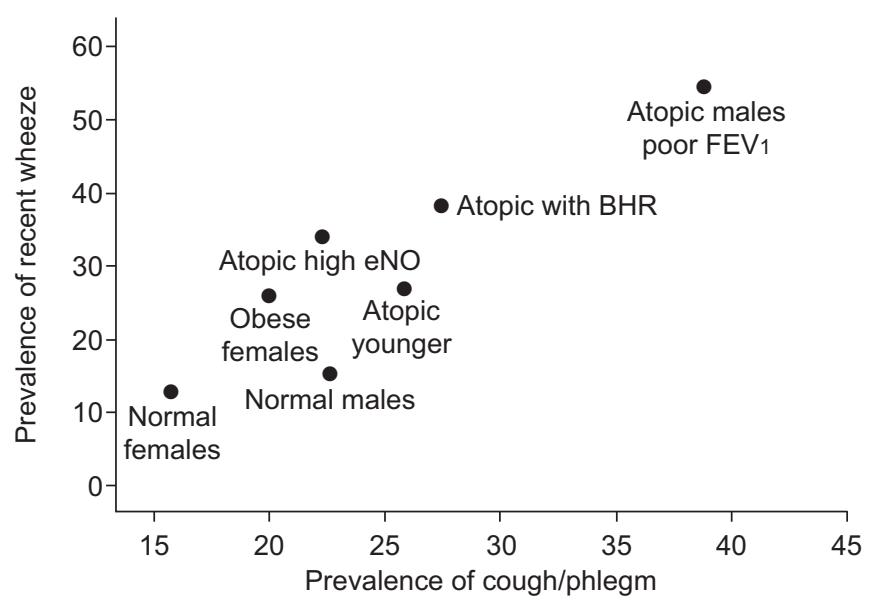

FIGURE 4. Patterns in prevalence of recent wheeze and cough/phlegm across clusters. BHR: bronchial hyperreactivity; FEV1: forced expiratory volume in $1 \mathrm{~s}$; eNO: exhaled nitric oxide. 
diagnosis, DDA, and current symptoms, cough and phlegm and recent wheeze, with our cluster groups.

It is now 100 yrs since the entity of "cardiac asthma" was separated from "bronchial asthma", when it became possible to differentiate between heart failure and airway narrowing as the predominant underlying physiological abnormality in most patients with variable wheeze and breathlessness. Over the intervening years, the adjective "bronchial" has been lost. However, different clinical patterns of bronchial asthma as diagnosed by a doctor (i.e. DDA) have long been recognised including: atopic and nonatopic asthma; childhood-onset and adult-onset asthma; reversible and irreversible asthma (as in asthma with COPD); neutrophilic and eosinophilic (and paucigranulocytic) asthma [8]; and steroid-responsive and steroid-nonresponsive asthma indicating an acceptance that bronchial asthma itself is a heterogeneous disease. Similarly, COPD and its synonyms are terms of clinical convenience because the causes and management of the different pathophysiological varieties are similar despite the knowledge that emphysema with primary loss of pulmonary elastic recoil is a different entity from irreversible intrinsic airway narrowing, as demonstrated by ColebAtch et al. [29] $>40$ yrs ago. The process of phenotypic reclassification needs to be extended, possibly with improved use of better/novel biomarkers [30], to further redefine the processes of airflow obstruction. Further dissection of airway phenotypes is needed to understand the molecular pathophysiology of airflow obstruction and allow coherent investigation of genotype-phenotype relationships. For example, a recent large genome-wide association study of DDA has shown that genetic associations of adult- and childhood-onset asthma differ [31].

Additional phenotypic analysis, such as assays of interleukin-6 or transforming growth factor- $\beta$ which appear to segregate with acquired and innate immune responses, may further advance the understanding of the airway phenotype-genotype relationships and may help to guide treatment with the advent of novel specific therapies in the future [32]. Similarly, more precise differentiation of the underlying physiological abnormalities or the morphological information provided by (highresolution) computed tomography could better guide therapy and genetic investigation (especially of COPD). This study of the general population confirms that the clinical entities of asthma and COPD are heterogeneous and suggests that the cluster approach may contribute towards disentangling the heterogeneity and might help to better identify specific risk factors and therapy.

\section{SUPPORT STATEMENT}

The 2005-2007 survey was funded by a project grant from the National Health and Medical Research Council (NHMRC) of Australia and the Sir Charles Gairdner Hospital Medical Research Foundation (Nedlands, Australia). A. James is supported by an NHMRC Practitioner Fellowship.

\section{STATEMENT OF INTEREST}

None declared.

\section{ACKNOWLEDGEMENTS}

The authors acknowledge the assistance of survey staff E. Inglis and R. Fleay (Busselton Population Medical Research Foundation, Nedlands, Australia), the local Busselton Health Study Action Committee and the many survey volunteers, as well as the long-standing support of the Busselton community for the surveys.

\section{REFERENCES}

1 Ciba Guest Symposium. Terminology, definitions and classification of chronic pulmonary emphysema and related conditions. A report of the conclusions of a Ciba Guest Symposium. Thorax 1959; 14: 286-299.

2 American Thoracic Society. Chronic Bronchitis, Asthma and Pulmonary Emphysema. A Statement by the Committee on Diagnostic Standards for Nontuberculous Respiratory Diseases. Am Rev Respir Dis 1962; 85: 762-768.

3 Hargreave FE, Nair P. The definition and diagnosis of asthma. Clin Exp Allergy 2009; 39: 1652-1658.

4 Pavord ID, Wardlaw AJ. The A to E of airway disease. Clin Exp Allergy 2010; 40: 62-67.

5 Wardlaw AJ, Silverman M, Siva R, et al. Multi-dimensional phenotyping: towards a new taxonomy for airway disease. Clin Exp Allergy 2005; 35: 1254-1262.

6 Boezen HM. Genome-wide association studies: what do they teach us about asthma and chronic obstructive pulmonary disease? Proc Am Thorac Soc 2009; 6: 701-703.

7 Haldar P, Brightling CE, Hargadon B, et al. Mepolizumab and exacerbations of refractory eosinophilic asthma. N Engl J Med 2009; 360: 973-984.

8 Gibson PG, Simpson JL, Saltos N. Heterogeneity of airway inflammation in persistent asthma: evidence of neutrophilic inflammation and increased sputum interleukin-8. Chest 2001; 119: 1329-1336.

9 Spycher BD, Silverman M, Kuehni CE. Phenotypes of childhood asthma: are they real? Clin Exp Allergy 2010; 40: 1130-1141.

10 Wenzel SE. Asthma: defining of the persistent adult phenotypes. Lancet 2006; 368: 804-813.

11 Haldar P, Pavord ID, Shaw DE, et al. Cluster analysis and clinical asthma phenotypes. Am J Respir Crit Care Med 2008; 178: 218-224.

12 Beasley R, Weatherall $\mathrm{M}$, Travers $\mathrm{J}$, et al. Time to define the disorders of the syndrome of COPD. Lancet 2009; 374: 670-672.

13 Spycher BD, Silverman M, Brooke AM, et al. Distinguishing phenotypes of childhood wheeze and cough using latent class analysis. Eur Respir J 2008; 31: 974-981.

14 Moore WC, Meyers DA, Wenzel SE, et al. Identification of asthma phenotypes using cluster analysis in the Severe Asthma Research Program. Am J Respir Crit Care Med 2010; 181: 315-323.

15 Pillai SG, Tang Y, van den Oord E, et al. Factor analysis in the Genetics of Asthma International Network family study identifies five major quantitative asthma phenotypes. Clin Exp Allergy 2008; 38: 421-429.

16 Weatherall $\mathrm{M}$, Shirtcliffe $\mathrm{P}$, Travers J, et al. Use of cluster analysis to define COPD phenotypes. Eur Respir J 2010; 36: 472-474.

17 Weatherall M, Travers J, Shirtcliffe PM, et al. Distinct clinical phenotypes of airways disease defined by cluster analysis. Eur Respir J 2009; 34: 812-818.

18 Lebowitz MD, Burrows B. Comparison of questionnaires: the BMRC and NHLI respiratory questionnaires and a new selfcompletion questionnaire. Am Rev Respir Dis 1976; 113: 627-635.

19 Hankinson JL, Odencrantz JR, Fedan KB. Spirometric reference values from a sample of the general US population. Am J Respir Crit Care Med 1999; 159: 179-187.

20 Miller MR, Hankinson J, Brusasco V, et al. Standardisation of spirometry. Eur Respir J 2005; 26: 319-338.

21 Buist AS, Vollmer WM, Sullivan SD, et al. The Burden of Obstructive Lung Disease Initiative (BOLD): rationale and design. COPD 2005; 2: 277-283.

22 Ryan G, Latimer KM, Dolovich J, et al. Bronchial responsiveness to histamine: relationship to diurnal variation of peak flow rate, improvement after bronchodilator, and airway calibre. Thorax 1982; 37: 423-429. 
23 Kharitonov SA, Yates D, Barnes PJ. Increased nitric oxide in exhaled air of normal human subjects with upper respiratory tract infections. Eur Respir J 1995; 8: 295-297.

24 Green RH, Brightling CE, Woltmann G, et al. Analysis of induced sputum in adults with asthma: identification of subgroup with isolated sputum neutrophilia and poor response to inhaled corticosteroids. Thorax 2002; 57: 875-879.

25 Simpson JL, Scott R, Boyle MJ, et al. Inflammatory subtypes in asthma: assessment and identification using induced sputum. Respirology 2006; 11: 54-61.

26 Moore WC, Meyers DA, Wenzel SE, et al. Identification of asthma phenotypes using cluster analysis in the Severe Asthma Research Program. Am J Respir Crit Care Med 2010; 181: 315-323.

27 Patel BD, Coxson HO, Pillai SG, et al. Airway wall thickening and emphysema show independent familial aggregation in chronic obstructive pulmonary disease. Am J Respir Crit Care Med 2008; 178: 500-505.

28 Ward JH. Hiererchical grouping to optimize and objective function. J Am Stat Assoc 1963; 58: 236-244.

29 Colebatch HJ, Finucane KE, Smith MM. Pulmonary conductance and elastic recoil relationships in asthma and emphysema. J Appl Physiol 1973; 34: 143-153.

30 Sin DD, Vestbo J. Biomarkers in chronic obstructive pulmonary disease. Proc Am Thorac Soc 2009; 6: 543-545.

31 Moffatt MF, Gut IG, Demenais F, et al. A large-scale, consortiumbased genomewide association study of asthma. $N$ Engl J Med 2010; 363: 1211-1221.

32 Travers J, Marsh S, Williams M, et al. External validity of randomised controlled trials in asthma: to whom do the results of the trials apply? Thorax 2007; 62: 219-223. 